\title{
Prediction model for Prolonged fever in patients with Mycoplasma pneumoniae pneumonia : A retrospective study of 716 pediatric patients
}

\section{Min Sik Jang}

Department of Pediatrics, Hallym University College of Medicine, Kangdong Sacred Heart Hospital Bit Kyeol Kim

Department of Pediatrics, Hallym University College of Medicine, Kangdong Sacred Heart Hospital Jihye Kim ( $\square$ jihyewiz17@gmail.com )

Department of Pediatrics, Hallym University College of Medicine, Kangdong Sacred Heart Hospital

\section{Research Article}

Keywords: Mycoplasma pneumoniae pneumonia, refractory, prediction

Posted Date: December 21st, 2020

DOl: https://doi.org/10.21203/rs.3.rs-127696/v1

License: () (1) This work is licensed under a Creative Commons Attribution 4.0 International License. Read Full License 


\section{Abstract}

Background: To identify patients with Mycoplasma pneumoniae pneumonia (MPP) with a risk of prolonged fever while on macrolides.

Methods: A retrospective study was performed with 716 children admitted for MPP. Refractory MPP (RMPP) was defined as fever persisting for $>72$ hours after macrolide antibiotics (RMPP-3) or when fever persisted for $>120$ hours (RMPP-5). Radiological data, laboratory data, and fever profiles were compared between the RMPP and non-RMPP groups. Fever profiles included the highest temperature, lowest temperature, and frequency of fever. Prediction models for RMPP were created using the logistic regression method and deep neural network. Their predictive values were compared using receiver operating characteristic curves.

Results: Overall, 716 patients were randomly divided into two groups: training and test cohorts for both RMPP-3 and RMPP-5. For the prediction of RMPP-3, a conventional logistic model with radiologic grouping showed increased sensitivity (63.3\%) than the model using laboratory values. Adding laboratory values in the prediction model using radiologic grouping did not contribute to a meaningful increase in sensitivity (64.6\%). For the prediction of RMPP-5, laboratory values or radiologic grouping showed lower sensitivities ranging from $12.9 \%-16.1 \%$. However, prediction models using predefined fever profiles showed significantly increased sensitivity for predicting RMPP-5, and neural network models using 12 sequential fever data showed a greatly increased sensitivity (64.5\%).

Conclusions: RMPP-5 could not be effectively predicted using initial laboratory and radiologic data, which were previously reported to be predictive. Further studies using advanced mathematical models, based on large-sized easily accessible clinical data, are anticipated for predicting RMPP.

\section{Background}

Mycoplasma pneumoniae (MP) infections are generally mild and self-limited. However, patients of every age can develop severe and progressive course during treatment with appropriate antibiotic therapy. ${ }^{1}$ The underlying mechanisms are unclear, but a direct microbe effect, macrolide resistance, and excessive immunological response of the host are commonly suggested. ${ }^{2,3}$ Macrolide antibiotics have been generally preferred as the first-choice agents for MP infections because secondary antibiotics such as tetracyclines and fluoroquinolones are not recommended because of the risk of severe adverse events, especially in pediatric patients.

Macrolide resistance rates have risen throughout the world and varies across countries. ${ }^{4-7}$ Although macrolides could be continued in the cases of mild to moderate infections irrespective of their resistance, replacement by alternative antibiotics or additional corticosteroids have been shown to improve radiological abnormalities and clinical symptoms. ${ }^{8,9}$ Additionally, the severity of disease is partially related to the degree to which the host immune response reacts to infection. The concept of immune- 
mediated lung disease provides a basis for consideration of immunomodulatory therapy in addition to conventional antimicrobial therapies for the management of MP infections. ${ }^{10}$

The appropriate time for alternative treatment is not clarified, but it depends on the physician's decision. Alternative treatments are delayed on some occasions owing to concerns regarding toxicities and adverse effects of secondary antibiotics or the possibility of blurred diagnosis caused by corticosteroids, leading to aggravation of the clinical course. At the initiation phase of macrolide therapy, physicians find it difficult to predict patients with a prolonged clinical course. Identifying these patients would help in providing them with timely treatment and mitigating their clinical course.

This study aims to identify the predictive factors of prolonged fever in patients with MP pneumonia with readily accessible clinical, laboratory, and radiological data and to develop a predictive model for these patients in whom timely initiation of secondary treatment options should be considered.

\section{Methods}

\section{Study design and ethical considerations}

The medical records of previously healthy children admitted for MP infection at our institution between January 2015 and December 2019 were retrospectively reviewed. All methods were carried out in accordance with the format recommended by the Strengthening the Reporting of Observational Studies in Epidemiology (STROBE) guidelines. The study protocol was approved by our institutional review board. The review board waived the requirement for informed consent for this study.

\section{Study patients}

All patients who had symptoms and signs indicative of pneumonia at admission, including fever $(\geq 38$ $\left.{ }^{\circ} \mathrm{C}\right)$, cough, and abnormal lung auscultation, were included. Empiric antibiotics were initially prescribed for these patients ( $\beta$-lactam agents and/or macrolides). Only patients initiated on a regimen with macrolides were included. Diagnosis of $M$. pneumoniae was confirmed by laboratory data and chest radiographs. A baseline blood sample and nasopharyngeal aspirate/swab (NPA) were collected for serological and microbiological testing. M. pneumoniae infection was confirmed using serologic testing and/or polymerase chain reaction (PCR) testing of the NPA. An enzyme immunoassay for IgM antibodies specific to M. pneumoniae (EIA, Bio-Rad Platelia ${ }^{\mathrm{TM}}$ M. pneumoniae IgM, California, USA) was performed with the initial blood samples according to the manufacturer's protocol.

We excluded patients with underlying diseases, patients who were treated for confirmed or suspected MP infection within the prior four weeks, patients with either positive IgM or PCR for MP but whose symptoms and radiographic findings were incompatible with pneumonia, patients treated with antiviral agents for proven influenza virus with fever onset within 72 hours, patients who received intravenous corticosteroids or changed to alternative antimicrobials (tetracyclines or fluoroquinolones) within 72 hours, and patients who were afebrile after admission. 
A case with persistent fever for $>72$ hours without improvement in radiological findings despite appropriate management with macrolides was defined as refractory M. pneumonia pneumonia (RMPP-3). Patients with persistent fever for $>120$ hours without improvement in radiological findings despite appropriate management were defined as RMPP-5.

\section{Grouping: training and test cohorts for RMPP-3 and RMPP-5}

Patients were randomly grouped into the training $(n=501)$ and test cohorts $(n=215)$ using simple random sampling without replacement (Figure 1). Each cohort was then categorized into the RMPP-3 group and non-RMPP-3 group based on their duration to defervescence. Defervescence was defined as maintenance of body temperature below $38^{\circ} \mathrm{C}$ for at least 24 hours. For the prediction analysis of patients with fever for $>120$ hours, the group randomization process was implemented again on the same cohort, after which each cohort was categorized into the RMPP- 5 and non-RMPP- 5 groups.

\section{Predictors: fever profiles}

The frequency of fever was defined as the number of peaks on the temperature curve. It was only counted when body temperature was $\geq 38.0^{\circ} \mathrm{C}$ and had increased $\geq 0.6^{\circ} \mathrm{C}$ within 4 hours. If the patient continued to have temperature changes of $<0.6^{\circ} \mathrm{C}$ but whose body temperature was $\geq 38.0^{\circ} \mathrm{C}$ during the 4-hour interval, it was counted as valid (continuous fever pattern).

\section{Predictors: clinical data}

Demographic and clinical information were collected in a standardized form by reviewing the electronic medical records. The following information was gathered: duration of fever (before and after hospitalization), total hospital days, and fever profile (highest body temperature, lowest body temperature, frequency of peak fever over $39^{\circ} \mathrm{C}$, frequency of peak fever over $40^{\circ} \mathrm{C}$, and total frequency of peak fever) extracted from 12 sequential fever data within 48 hours. These fever profiles were only included in the analysis for the prediction of prolonged fever over 120 hours (RMPP-5).

\section{Predictors: laboratory data}

Tests for complete blood count (CBC), serum aminotransferase, erythrocyte sedimentation rate (ESR), Creactive protein (CRP), lactate dehydrogenase (LDH), procalcitonin, and blood cultures were performed. FilmArray multiplex PCR system (Biomérieux, USA) was used for detecting common respiratory tract virus antigens.

\section{Predictors: radiologic data}

Chest radiographs were reviewed independently by two experienced radiologists. They were blinded to the clinical data and original radiographic interpretations. Radiological findings at admission were categorized into four groups: group 1, patients with parahilar peribronchial opacification or diffuse 
interstitial infiltration; group 2, patients with reticular, nodular, or reticulonodular densities; group 3, patients with segmental or lobar consolidation in a single lobe with or without pleural effusion of 1/4$1 / 2$ in the decubitus position; and group 4, patients with lobar consolidation in 2 or more lobes and/or pleural effusion of more than $1 / 2$ in the decubitus position. The images were interpreted and compared by two radiologists to reach a consensus.

\section{Statistical analyses}

Continuous variables were presented as mean \pm standard deviation and were compared using an independent $t$ test. Categorical variables were presented as frequency (\%) and were compared using the Pearson chi-squared test or Fisher's exact test (Table 1 and 2).

Table 1. Initial variables of the whole cohort (fever $>72$ hours) 


\begin{tabular}{|c|c|c|c|c|c|c|c|}
\hline & \multicolumn{3}{|c|}{ Training cohort $(\mathrm{n}=501)$} & \multicolumn{3}{|c|}{ Test cohort $(\mathrm{n}=215)$} \\
\hline & & $\begin{array}{c}\text { Fever } \\
\geq 72 \\
\text { hours }\end{array}$ & $\begin{array}{c}\text { Fever }<72 \\
\text { hours }\end{array}$ & $p$-value & $\begin{array}{c}\text { Fever } \\
\geq 72 \\
\text { hours }\end{array}$ & $\begin{array}{c}\begin{array}{c}\text { Fever } \\
<72 \\
\text { hours }\end{array}\end{array}$ & $p$-value \\
\hline \multicolumn{2}{|l|}{$\begin{array}{l}\text { Number of patients } \\
(n, \%)\end{array}$} & $\begin{array}{c}163 \\
(32.5)\end{array}$ & $\begin{array}{c}338 \\
(67.5)\end{array}$ & & $\begin{array}{c}79 \\
(36.7)\end{array}$ & $\begin{array}{c}136 \\
(63.3)\end{array}$ & \\
\hline \multicolumn{2}{|l|}{ Age, mean (years) } & $5.9 \pm 2.8$ & $5.5 \pm 3.2$ & 0.140 & $5.8 \pm 2.5$ & $5.3 \pm 3.1$ & 0.273 \\
\hline \multicolumn{2}{|l|}{ Sex ratio (F:M) } & 1.3 & 1.0 & 0.124 & 1.7 & 0.7 & 0.002 \\
\hline \multirow{3}{*}{$\begin{array}{l}\text { Duration of fever } \\
\text { (days) }\end{array}$} & At admission & $4.8 \pm 1.8$ & $5.1 \pm 2.2$ & 0.057 & $4.9 \pm 1.7$ & $4.9 \pm 2.0$ & 0.960 \\
\hline & After admission & $4.8 \pm 3.4$ & $1.3 \pm 1.0$ & $<0.001$ & $4.9 \pm 3.3$ & $1.3 \pm 0.9$ & $<0.001$ \\
\hline & Total & $9.4 \pm 3.3$ & $6.5 \pm 2.3$ & $<0.001$ & $9.5 \pm 3.0$ & $\begin{array}{l}6.3 \pm \\
2.1\end{array}$ & $<0.001$ \\
\hline \multicolumn{2}{|l|}{$\begin{array}{l}\text { Total hospital days } \\
\text { (days) }\end{array}$} & $9.2 \pm 3.5$ & $5.4 \pm 1.6$ & $<0.001$ & $9.4 \pm 3.6$ & $5.5 \pm 1.8$ & $<0.001$ \\
\hline \multirow{9}{*}{$\begin{array}{l}\text { Initial inflammatory } \\
\text { markers }\end{array}$} & $\mathrm{WBC} \times 10^{3} / \mu \mathrm{L}$ & $7.6 \pm 3.5$ & $9.6 \pm 7.0$ & 0.001 & $7.5 \pm 3.2$ & $9.7 \pm 5.5$ & $<0.001$ \\
\hline & Neutrophils (\%) & $\begin{array}{c}67.0 \pm \\
10.7\end{array}$ & $\begin{array}{c}63.0 \pm \\
14.2\end{array}$ & 0.001 & $\begin{array}{c}68.0 \pm \\
10.8\end{array}$ & $\begin{array}{c}61.9 \pm \\
14.2\end{array}$ & 0.001 \\
\hline & $\begin{array}{l}\text { Absolute neutrophil } \\
\text { count } \times 10^{3} / \mu \mathrm{L}\end{array}$ & $5.2 \pm 2.9$ & $6.2 \pm 5.3$ & 0.003 & $5.1 \pm 2.4$ & $6.2 \pm 4.8$ & 0.031 \\
\hline & Lymphocytes (\%) & $\begin{array}{c}23.2 \pm \\
8.4\end{array}$ & $\begin{array}{c}26.6 \pm \\
12.4\end{array}$ & $<0.001$ & $\begin{array}{c}22.9 \pm \\
8.4\end{array}$ & $\begin{array}{c}27.7 \pm \\
12.8\end{array}$ & 0.001 \\
\hline & $\begin{array}{l}\text { Absolute lymphocyte } \\
\text { count } \times 10^{3} / \mu \mathrm{L}\end{array}$ & $1.1 \pm 0.5$ & $1.4 \pm 1.1$ & $<0.001$ & $1.1 \pm 0.6$ & $1.4 \pm 0.7$ & 0.001 \\
\hline & Platelet $\times 10^{3} / \mu \mathrm{L}$ & $\begin{array}{l}242.0 \pm \\
75.9\end{array}$ & $\begin{array}{c}304.9 \pm \\
100.4\end{array}$ & $<0.001$ & $\begin{array}{c}234.9 \pm \\
71.1\end{array}$ & $\begin{array}{c}308.6 \pm \\
101.1\end{array}$ & $<0.001$ \\
\hline & ESR $(\mathrm{mm} / \mathrm{hr})$ & $\begin{array}{c}38.3 \pm \\
18.4\end{array}$ & $\begin{array}{c}35.9 \pm \\
18.5\end{array}$ & 0.178 & $\begin{array}{c}37.4 \pm \\
17.3\end{array}$ & $\begin{array}{c}32.5 \pm \\
17.4\end{array}$ & $<0.001$ \\
\hline & $\mathrm{CRP}(\mathrm{mg} / \mathrm{L})$ & $\begin{array}{l}49.2 \pm \\
45.6\end{array}$ & $\begin{array}{l}24.7 \pm \\
30.6\end{array}$ & $<0.001$ & $\begin{array}{c}57.7 \pm \\
53.9\end{array}$ & $\begin{array}{c}24.1 \pm \\
27.7\end{array}$ & $<0.001$ \\
\hline & Procalcitonin $(\mathrm{ng} / \mathrm{mL})$ & $0.6 \pm 1.2$ & $0.7 \pm 2.4$ & 0.700 & $0.5 \pm 0.7$ & $0.3 \pm 0.6$ & 0.169 \\
\hline \multirow[t]{3}{*}{ Other laboratory data } & LDH (IU/L) & $\begin{array}{c}359.8 \pm \\
107.4\end{array}$ & $\begin{array}{l}326.2 \pm \\
73.1\end{array}$ & $<0.001$ & $\begin{array}{l}372.6 \pm \\
132.8\end{array}$ & $\begin{array}{l}332.7 \pm \\
90.7\end{array}$ & 0.019 \\
\hline & AST (IU/L) & $\begin{array}{c}40.3 \pm \\
17.9\end{array}$ & $\begin{array}{l}36.2 \pm \\
29.3\end{array}$ & 0.104 & $\begin{array}{c}39.4 \pm \\
14.9\end{array}$ & $\begin{array}{l}41.5 \pm \\
48.5\end{array}$ & 0.701 \\
\hline & ALT (IU/L) & $\begin{array}{c}19.6 \pm \\
13.3\end{array}$ & $\begin{array}{c}22.6 \pm \\
64.9\end{array}$ & 0.556 & $\begin{array}{c}15.9 \pm \\
7.3\end{array}$ & $\begin{array}{c}29.2 \pm \\
77.9\end{array}$ & 0.051 \\
\hline \multicolumn{2}{|l|}{$\begin{array}{l}\text { Concurrent } \\
\text { respiratory virus ( } n \text {, } \\
\%)\end{array}$} & $\begin{array}{c}36 / 140 \\
(25.7)\end{array}$ & $\begin{array}{c}95 / 285 \\
(33.3)\end{array}$ & 0.110 & $\begin{array}{l}18 / 64 \\
(28.1)\end{array}$ & $\begin{array}{c}39 / 117 \\
(33.3)\end{array}$ & 0.471 \\
\hline \multicolumn{2}{|l|}{$\begin{array}{l}\text { Oxygen requirement } \\
(n, \%)\end{array}$} & $5(3.1)$ & $8(2.4)$ & 0.765 & $3(3.8)$ & $2(1.5)$ & 0.359 \\
\hline \multirow[t]{4}{*}{$\begin{array}{l}\text { Radiologic grouping } \\
(n, \%)\end{array}$} & Group 1 & $13(8.0)$ & $\begin{array}{c}108 \\
(32.0)\end{array}$ & $<0.001$ & $7(8.9)$ & $44(32.4)$ & $<0.001$ \\
\hline & Group 2 & $54(33.1)$ & $\begin{array}{c}161 \\
(47.6)\end{array}$ & & $\begin{array}{c}22 \\
(27.8)\end{array}$ & $67(49.3)$ & \\
\hline & Group 3 & $85(52.1)$ & $68(20.1)$ & & $\begin{array}{c}41 \\
(51.9)\end{array}$ & $24(17.6)$ & \\
\hline & Group 4 & $11(6.7)$ & $1(0.3)$ & & $9(11.4)$ & $1(0.7)$ & \\
\hline \multicolumn{2}{|l|}{ Pleural effusion $(n, \%)$} & 34 (20.9) & $20(5.9)$ & $<0.001$ & $\begin{array}{c}18 \\
(22.8)\end{array}$ & $6(4.4)$ & $<0.001$ \\
\hline \multicolumn{2}{|c|}{$\begin{array}{l}\text { Radiologic aggravation on the } 3^{\text {rd }} \text { or } 4^{\text {th }} \text { hospital } \\
\text { day }(n, \%)\end{array}$} & $\begin{array}{c}121 \\
(74.2)\end{array}$ & $29(8.6)$ & $<0.001$ & $\begin{array}{c}61 \\
(77.2)\end{array}$ & $15(11.0)$ & $<0.001$ \\
\hline
\end{tabular}


Data are presented as the mean \pm standard deviation

ESR: Erythrocyte sedimentation rate

CRP: C-reactive protein

LDH: Lactate dehydrogenase

AST: Aspartate aminotransferase

ALT: Alanine aminotransferase

WBC: White blood cell

Table 2. Initial variables of the whole cohort (fever $>120$ hours) 


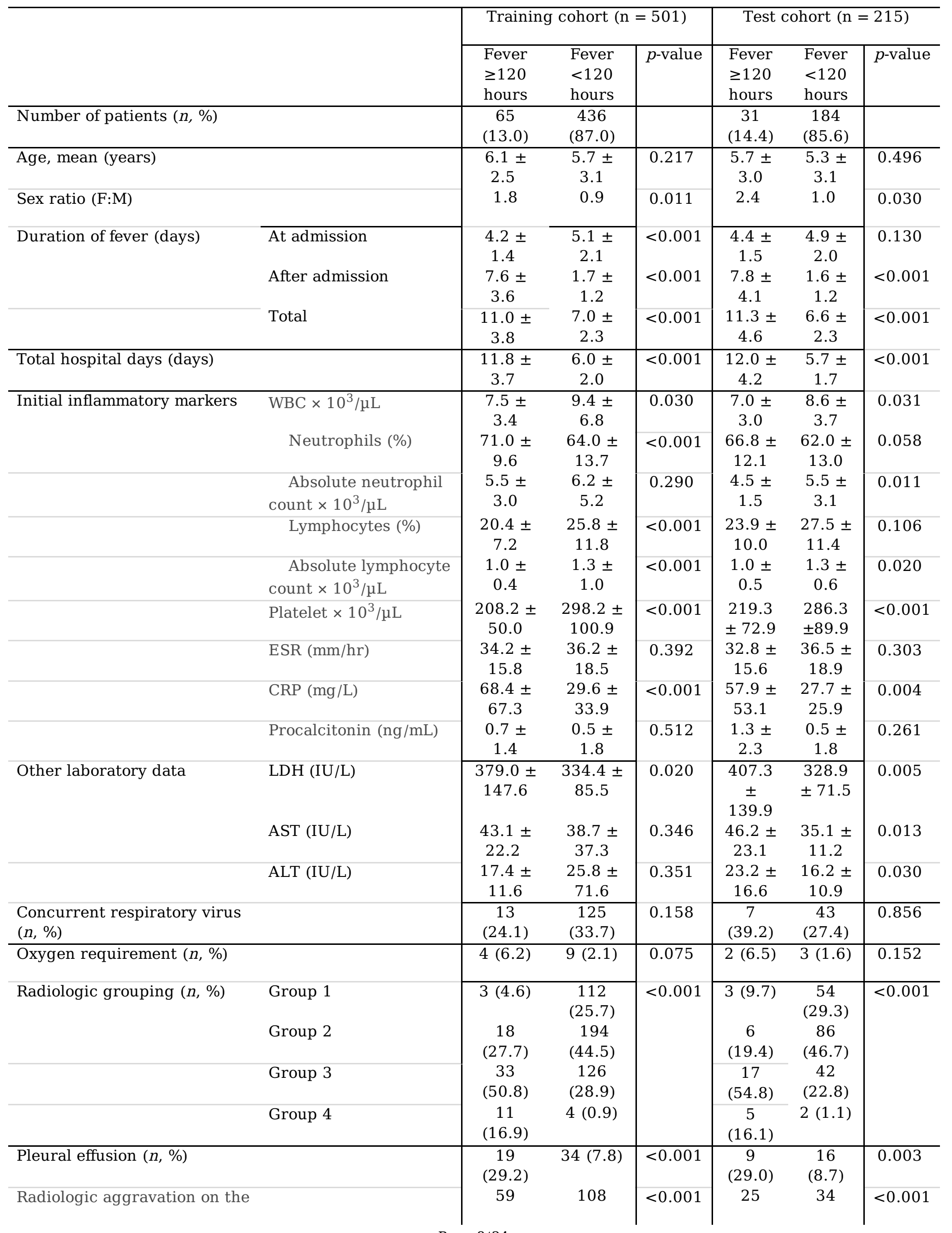




\begin{tabular}{|c|c|c|c|c|c|c|c|}
\hline $3^{\text {rd }}$ or $4^{\text {th }}$ hospital day $(n, \%)$ & & $(90.8)$ & $(24.8)$ & & $(80.6)$ & $(18.5)$ & \\
\hline \multirow[t]{6}{*}{ Fever profiles } & $\begin{array}{l}\text { Highest temperature } \\
\left({ }^{\circ} \mathrm{C}\right)\end{array}$ & $\begin{array}{c}39.6 \pm \\
0.6\end{array}$ & $\begin{array}{c}38.7 \pm \\
0.7\end{array}$ & $<0.001$ & $\begin{array}{c}39.4 \pm \\
0.6\end{array}$ & $\begin{array}{c}38.7 \pm \\
0.7\end{array}$ & $<0.001$ \\
\hline & $\begin{array}{l}\text { Lowest temperature } \\
\left({ }^{\circ} \mathrm{C}\right)\end{array}$ & $\begin{array}{c}37.0 \pm \\
0.3\end{array}$ & $\begin{array}{c}36.6 \pm \\
0.3\end{array}$ & $<0.001$ & $\begin{array}{c}36.9 \pm \\
0.3\end{array}$ & $\begin{array}{c}36.6 \pm \\
0.3\end{array}$ & $<0.001$ \\
\hline & $\begin{array}{l}\text { Frequency of fever } \\
>39^{\circ} \mathrm{C} \text { within } 48 \text { hours } \\
\text { (n) }\end{array}$ & $\begin{array}{c}3.3 \pm \\
2.8\end{array}$ & $\begin{array}{c}0.7 \pm \\
1.4\end{array}$ & $<0.001$ & $\begin{array}{c}2.9 \pm \\
1.7\end{array}$ & $\begin{array}{l}0.6 \pm \\
1.2\end{array}$ & $<0.001$ \\
\hline & $\begin{array}{l}\text { Frequency of fever } \\
>40^{\circ} \mathrm{C} \text { within } 48 \text { hours } \\
\text { (n) }\end{array}$ & $\begin{array}{l}0.5 \pm \\
1.0\end{array}$ & $\begin{array}{c}0.1 \pm \\
0.3\end{array}$ & $<0.001$ & $\begin{array}{c}0.3 \pm \\
0.6\end{array}$ & $\begin{array}{c}0.0 \pm \\
0.2\end{array}$ & 0.070 \\
\hline & $\begin{array}{l}\text { Frequency of peak } \\
\text { fever within } 24 \text { hours } \\
\text { (n) }\end{array}$ & $\begin{array}{l}4.7 \pm \\
1.2\end{array}$ & $\begin{array}{c}2.9 \pm \\
1.4\end{array}$ & $<0.001$ & $\begin{array}{c}4.6 \pm \\
1.0\end{array}$ & $\begin{array}{c}2.8 \pm \\
1.3\end{array}$ & $<0.001$ \\
\hline & $\begin{array}{l}\text { Frequency of peak } \\
\text { fever within } 48 \text { hours } \\
\text { (n) }\end{array}$ & $\begin{array}{c}8.7 \pm \\
1.7\end{array}$ & $\begin{array}{c}4.6 \pm \\
2.7\end{array}$ & $<0.001$ & $\begin{array}{c}8.6 \pm \\
1.7\end{array}$ & $\begin{array}{l}4.5 \pm \\
2.6\end{array}$ & $<0.001$ \\
\hline \multirow[t]{12}{*}{$\begin{array}{l}12 \text { sequential body } \\
\text { temperatures }\end{array}$} & Initial $\left({ }^{\circ} \mathrm{C}\right)$ & $\begin{array}{c}38.6 \pm \\
0.9\end{array}$ & $\begin{array}{c}37.9 \pm \\
0.9\end{array}$ & $<0.001$ & $\begin{array}{c}38.2 \pm \\
0.9\end{array}$ & $\begin{array}{c}37.9 \pm \\
0.9\end{array}$ & 0.100 \\
\hline & after 4 hours $\left({ }^{\circ} \mathrm{C}\right)$ & $\begin{array}{c}38.1 \pm \\
0.9\end{array}$ & $\begin{array}{c}37.6 \pm \\
0.8\end{array}$ & $<0.001$ & $\begin{array}{c}38.0 \pm \\
0.9\end{array}$ & $\begin{array}{c}37.6 \pm \\
0.7\end{array}$ & 0.008 \\
\hline & after 8 hours $\left({ }^{\circ} \mathrm{C}\right)$ & $\begin{array}{l}37.9 \pm \\
0.8\end{array}$ & $\begin{array}{c}37.4 \pm \\
0.9\end{array}$ & $<0.001$ & $\begin{array}{c}38.0 \pm \\
1.1\end{array}$ & $\begin{array}{l}37.5 \pm \\
0.8\end{array}$ & 0.016 \\
\hline & after 12 hours $\left({ }^{\circ} \mathrm{C}\right)$ & $\begin{array}{c}37.9 \pm \\
0.9\end{array}$ & $\begin{array}{c}37.4 \pm \\
0.9\end{array}$ & $<0.001$ & $\begin{array}{c}38.0 \pm \\
1.0\end{array}$ & $\begin{array}{c}37.4 \pm \\
0.8\end{array}$ & $<0.001$ \\
\hline & after 16 hours $\left({ }^{\circ} \mathrm{C}\right)$ & $\begin{array}{c}38.0 \pm \\
1.0\end{array}$ & $\begin{array}{c}37.4 \pm \\
0.8\end{array}$ & $<0.001$ & $\begin{array}{c}38.0 \pm \\
0.9\end{array}$ & $\begin{array}{c}37.3 \pm \\
0.8\end{array}$ & $<0.001$ \\
\hline & after 20 hours $\left({ }^{\circ} \mathrm{C}\right)$ & $\begin{array}{c}38.2 \pm \\
0.9\end{array}$ & $\begin{array}{c}37.4 \pm \\
0.7\end{array}$ & $<0.001$ & $\begin{array}{c}38.2 \pm \\
0.6\end{array}$ & $\begin{array}{c}37.4 \pm \\
0.8\end{array}$ & $<0.001$ \\
\hline & after 24 hours $\left({ }^{\circ} \mathrm{C}\right)$ & $\begin{array}{c}38.0 \pm \\
0.6\end{array}$ & $\begin{array}{c}37.4 \pm \\
0.7\end{array}$ & $<0.001$ & $\begin{array}{l}38.2 \pm \\
0.6\end{array}$ & $\begin{array}{c}37.5 \pm \\
0.7\end{array}$ & $<0.001$ \\
\hline & after 28 hours $\left({ }^{\circ} \mathrm{C}\right)$ & $\begin{array}{c}38.3 \pm \\
1.0\end{array}$ & $\begin{array}{c}37.5 \pm \\
0.8\end{array}$ & $<0.001$ & $\begin{array}{c}37.9 \pm \\
0.7\end{array}$ & $\begin{array}{c}37.4 \pm \\
0.7\end{array}$ & $<0.001$ \\
\hline & after 32 hours $\left({ }^{\circ} \mathrm{C}\right)$ & $\begin{array}{c}37.7 \pm \\
1.0\end{array}$ & $\begin{array}{c}37.0 \pm \\
2.4\end{array}$ & 0.040 & $\begin{array}{c}37.9 \pm \\
1.0\end{array}$ & $\begin{array}{c}37.2 \pm \\
0.7\end{array}$ & 0.001 \\
\hline & after 36 hours $\left({ }^{\circ} \mathrm{C}\right)$ & $\begin{array}{c}37.9 \pm \\
0.9\end{array}$ & $\begin{array}{c}37.1 \pm \\
0.7\end{array}$ & $<0.001$ & $\begin{array}{c}37.6 \pm \\
0.7\end{array}$ & $\begin{array}{c}37.1 \pm \\
0.8\end{array}$ & 0.001 \\
\hline & after 40 hours $\left({ }^{\circ} \mathrm{C}\right)$ & $\begin{array}{c}38.1 \pm \\
0.9\end{array}$ & $\begin{array}{c}37.1 \pm \\
0.7\end{array}$ & $<0.001$ & $\begin{array}{c}37.9 \pm \\
0.9\end{array}$ & $\begin{array}{c}37.0 \pm \\
0.7\end{array}$ & $<0.001$ \\
\hline & after 44 hours $\left({ }^{\circ} \mathrm{C}\right)$ & $\begin{array}{c}38.1 \pm \\
0.8\end{array}$ & $\begin{array}{c}37.1 \pm \\
0.7\end{array}$ & $<0.001$ & $\begin{array}{c}37.8 \pm \\
0.8\end{array}$ & $\begin{array}{c}37.1 \pm \\
0.7\end{array}$ & $<0.001$ \\
\hline
\end{tabular}

Data are presented as the mean \pm standard deviation

ESR: Erythrocyte sedimentation rate

CRP: C-reactive protein

LDH: Lactate dehydrogenase

AST: Aspartate aminotransferase

ALT: Alanine aminotransferase

WBC: White blood cell

Based on the data from the training cohorts, the univariate logistic regression analysis was performed for identifying significant independent predictors for RMPP-3 or RMPP-5 (Tables 3 and 4). With the significant predictors, stepwise multivariate logistic regression analysis was performed for creating 
conventional prediction models. To reflect the 12 sequential fever data on the prediction models effectively, a deep neural network (DNN) model was additionally created. DNN included two hidden layers. A dropout layer was used after the first hidden layer to prevent overfitting. For hyperparameter optimization, $20 \%$ of the training cohort patients were assigned to the validation cohort. Optimization was performed using the Adam method, and model loss was calculated through binary cross-entropy.

Calculations to determine the optimal number of layers and neurons for all DNNs were performed. For each combination of layers and hidden units, hyperparameters for obtaining the best performance for the combination were optimized.

Table 3. Conventional logistic model using data of the training cohort: RMPP-3

\begin{tabular}{|c|c|c|c|c|c|c|c|}
\hline & \multicolumn{3}{|c|}{ Univariate } & \multicolumn{3}{|c|}{ Multivariate } \\
\hline & & $\begin{array}{l}\text { Odds } \\
\text { ratio }\end{array}$ & $\begin{array}{l}\text { 95\% confidence } \\
\text { interval }\end{array}$ & $\begin{array}{c}p- \\
\text { value }\end{array}$ & $\begin{array}{l}\text { Odds } \\
\text { ratio }\end{array}$ & $\begin{array}{l}\text { 95\% confidence } \\
\text { interval }\end{array}$ & $\begin{array}{c}p- \\
\text { value }\end{array}$ \\
\hline Age (years) & & 1.046 & $(0.985,1.110)$ & 0.140 & & & \\
\hline Sex $(F: M)$ & & 1.345 & $(0.922,1.955)$ & 0.125 & & & \\
\hline $\mathrm{WBC} \times 10^{3} / \mu \mathrm{L}$ & & 1.000 & $(1.000,1.000)$ & $<0.001$ & \multirow{8}{*}{0.991} & \multirow{8}{*}{$(0.988,0.994)$} & \\
\hline Neutrophils (\%) & & 1.024 & $(1.009,1.039)$ & 0.002 & & & \\
\hline $\begin{array}{l}\text { Absolute neutrophil count } \\
\times 10^{3} / \mu \mathrm{L}\end{array}$ & & 1.000 & $(1.000,1.000)$ & 0.018 & & & \\
\hline Lymphocytes (\%) & & 0.972 & $(0.955,0.990)$ & 0.002 & & & \\
\hline $\begin{array}{l}\text { Absolute lymphocyte } \\
\text { count } \times 10^{3} / \mu \mathrm{L}\end{array}$ & & 0.999 & $(0.999,1.000)$ & $<0.001$ & & & \\
\hline Hemoglobin (g/dL) & & 0.946 & $(0.772,1.159)$ & 0.590 & & & \\
\hline Platelets $\times 10^{3} / \mu \mathrm{L}$ & & 0.991 & $(0.989,0.994)$ & $<0.001$ & & & $<0.001$ \\
\hline ESR (mm/hr) & & 1.007 & $(0.997,1.017)$ & 0.179 & & & \\
\hline $\mathrm{CRP}(\mathrm{mg} / \mathrm{L})$ & & 1.019 & $(1.013,1.026)$ & $<0.001$ & 1.014 & $(1.008,1.021)$ & $<0.001$ \\
\hline Procalcitonin (ng/mL) & & 0.973 & $(0.845,1.120)$ & 0.700 & \multirow{4}{*}{1.006} & \multirow{4}{*}{$(1.003,1.009)$} & \\
\hline LDH (IU/L) & & 1.004 & $(1.002,1.007)$ & $<0.001$ & & & $<0.001$ \\
\hline AST (IU/L) & & 1.006 & $(0.998,1.013)$ & 0.126 & & & \\
\hline $\operatorname{ALT}(\mathrm{IU} / \mathrm{L})$ & & 0.999 & $(0.995,1.003)$ & 0.563 & & & \\
\hline $\begin{array}{l}\text { Concurrent respiratory } \\
\text { virus }\end{array}$ & & 1.444 & $(0.919,2.270)$ & 0.111 & & & \\
\hline Oxygen requirement & & 0.766 & $(0.247,2.379)$ & 0.645 & & & \\
\hline \multirow[t]{4}{*}{ Radiologic grouping } & $\begin{array}{l}\text { Group } \\
1\end{array}$ & - & - & $\begin{array}{c}<0.001 \\
-\end{array}$ & \multirow[b]{2}{*}{2.408} & \multirow[b]{2}{*}{$(1.209,4.796)$} & $<0.001$ \\
\hline & $\begin{array}{l}\text { Group } \\
2\end{array}$ & 2.786 & $(1.451,5.352)$ & 0.002 & & & 0.012 \\
\hline & $\begin{array}{l}\text { Group } \\
3\end{array}$ & 10.385 & $(5.379,20.049)$ & $<0.001$ & 8.080 & $(4.005,16.302)$ & $<0.001$ \\
\hline & $\begin{array}{l}\text { Group } \\
4\end{array}$ & 91.385 & $\begin{array}{l}(10.899, \\
766.257)\end{array}$ & $<0.001$ & 11.827 & $(1.181,118.493)$ & 0.036 \\
\hline Pleural effusion & & 4.191 & $(2.325,7.552)$ & $<0.001$ & & & \\
\hline
\end{tabular}

RMPP-3 : Refractory Mycoplasma pneumoniae pneumonia with fever for $\geq 72$ hours

ESR: Erythrocyte sedimentation rate

CRP: C-reactive protein

LDH: Lactate dehydrogenase

AST: Aspartate aminotransferase 
ALT: Alanine aminotransferase

Table 4. Conventional logistic model using data of the training cohort: RMPP-5 


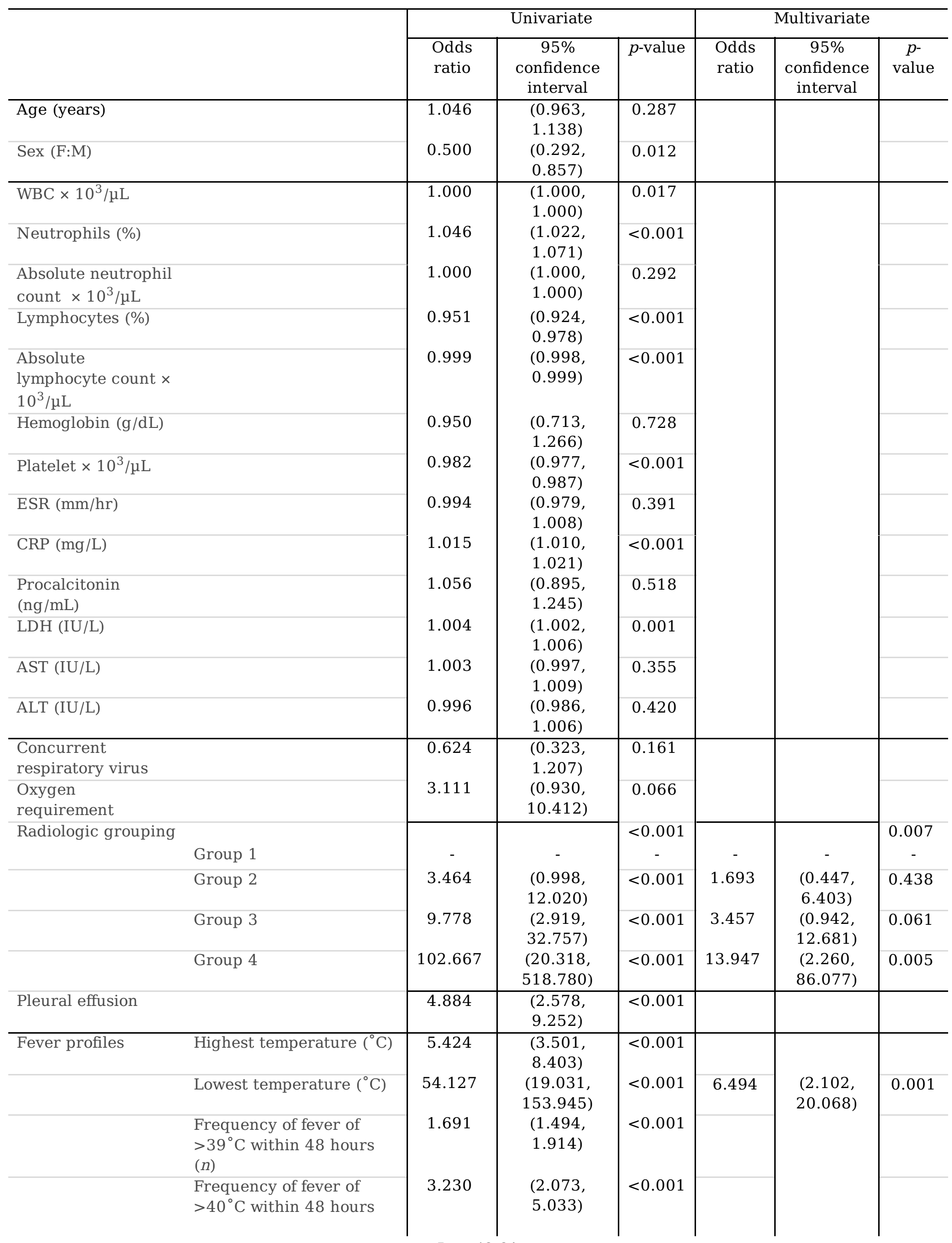




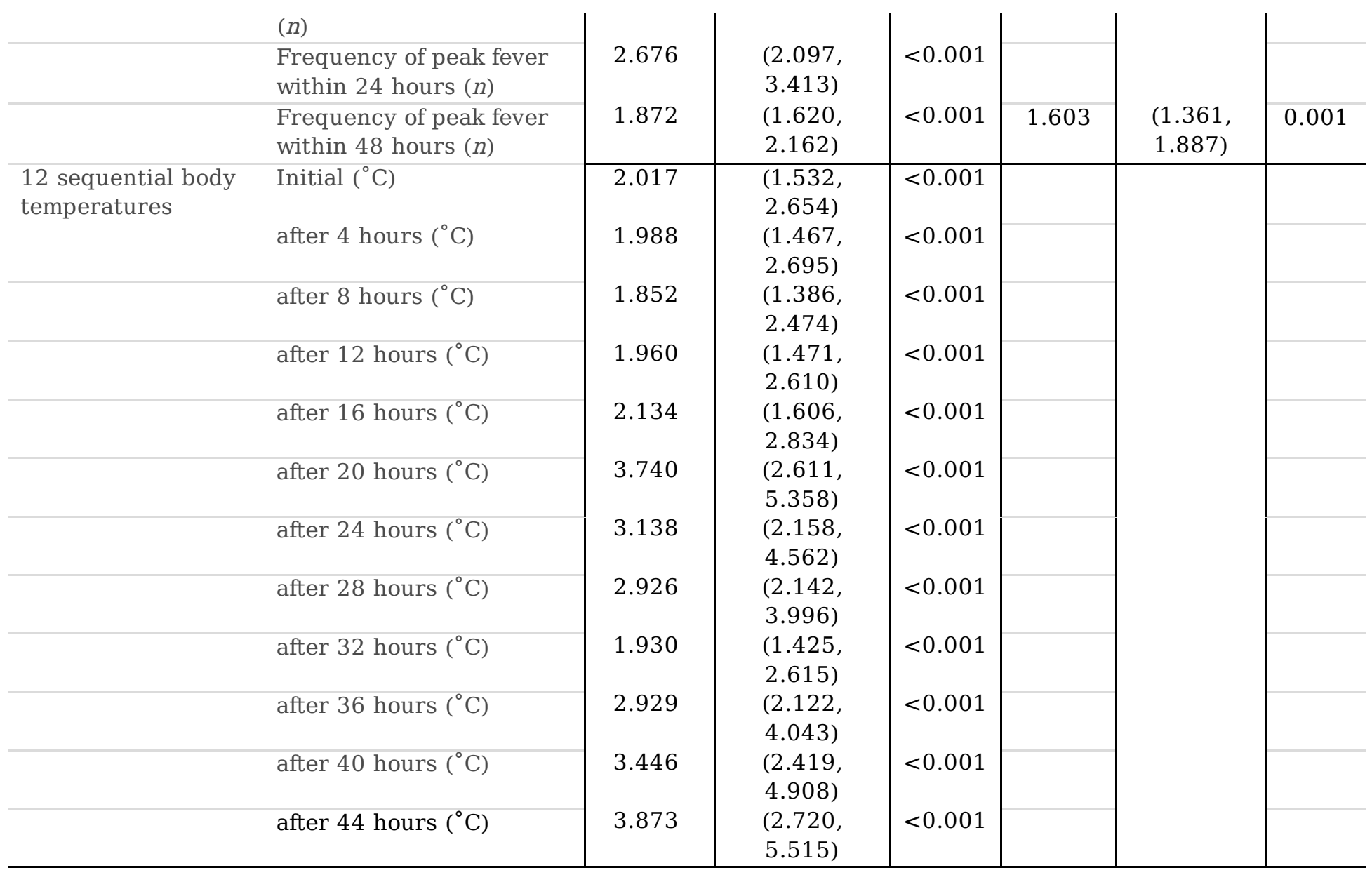

RMPP-5 : Refractory Mycoplasma pneumoniae pneumonia with fever for $\geq 120$ hours

ESR: Erythrocyte sedimentation rate

CRP: C-reactive protein

LDH: Lactate dehydrogenase

AST: Aspartate aminotransferase

ALT: Alanine aminotransferase

The prediction power of the conventional logistic prediction model and the DNN model was evaluated in the test cohorts using receiver operating characteristic (ROC) curves (Tables 5 and 6). Logistic regression analysis was performed using SPSS 25 (IBM Corp., Armonk, NY, USA). DNN models were developed using Python 3.7 (open-source projects) with Anaconda 4.7.12, and TensorFlow 2.0.

Table 5. Prediction of RMPP-3 (fever $>72$ hours) in the test cohort. 


\begin{tabular}{|c|c|c|c|c|c|c|c|c|c|}
\hline $\begin{array}{l}\text { Prediction } \\
\text { method }\end{array}$ & $\begin{array}{l}\text { Used } \\
\text { variables }\end{array}$ & $p$-value & $\begin{array}{c}\text { AUC } \\
\text { area } \\
(95 \% \\
\text { CI })\end{array}$ & Sensitivity & Specificity & $\begin{array}{c}\text { PPV } \\
\text { (Precision) }\end{array}$ & NPV & $\begin{array}{c}\text { Overall } \\
\text { accuracy }\end{array}$ & $\begin{array}{l}\text { Youden } \\
\text { Index }\end{array}$ \\
\hline $\begin{array}{l}\text { Conventional } \\
\text { logistic } \\
\text { model }\end{array}$ & CRP, LDH & $<0.001$ & $\begin{array}{c}0.642 \\
(0.562, \\
0.723)\end{array}$ & $32.9 \%$ & $95.2 \%$ & $81.3 \%$ & $69.4 \%$ & $71.2 \%$ & 0.281 \\
\hline $\begin{array}{l}\text { Conventional } \\
\text { logistic } \\
\text { model }\end{array}$ & $\begin{array}{l}\text { Radiologic } \\
\text { grouping }\end{array}$ & $<0.001$ & $\begin{array}{c}0.725 \\
(0.651 \\
0.798)\end{array}$ & $63.3 \%$ & $81.6 \%$ & $66.7 \%$ & $79.3 \%$ & $74.9 \%$ & 0.449 \\
\hline $\begin{array}{l}\text { Conventional } \\
\text { logistic } \\
\text { model }\end{array}$ & $\begin{array}{l}\text { Platelets, } \\
\text { CRP, LDH, } \\
\text { radiologic } \\
\text { grouping }\end{array}$ & $<0.001$ & $\begin{array}{c}0.775 \\
(0.704 \\
0.846)\end{array}$ & $64.6 \%$ & $90.4 \%$ & $79.7 \%$ & $81.5 \%$ & $80.9 \%$ & 0.550 \\
\hline
\end{tabular}

Table 6. Prediction of RMPP-5 (fever $>120$ hours) in the test cohort.

\begin{tabular}{|c|c|c|c|c|c|c|c|c|c|}
\hline $\begin{array}{l}\text { Prediction } \\
\text { method }\end{array}$ & $\begin{array}{l}\text { Used } \\
\text { variables }\end{array}$ & $p$-value & $\begin{array}{l}\text { AUC } \\
\text { area } \\
(95 \% \\
\text { CI })\end{array}$ & sensitivity & specificity & $\begin{array}{c}\text { PPV } \\
\text { (Precision) }\end{array}$ & NPV & $\begin{array}{c}\text { Overall } \\
\text { accuracy }\end{array}$ & $\begin{array}{l}\text { Youden } \\
\text { Index }\end{array}$ \\
\hline $\begin{array}{l}\text { Conventional } \\
\text { logistic } \\
\text { model }\end{array}$ & $\begin{array}{l}\text { ALC, CRP, } \\
\text { LDH }\end{array}$ & 0.251 & $\begin{array}{c}0.565 \\
(0.447, \\
0.682)\end{array}$ & $12.9 \%$ & $100.0 \%$ & $100.0 \%$ & $87.1 \%$ & $87.4 \%$ & 0.129 \\
\hline $\begin{array}{l}\text { Conventional } \\
\text { logistic } \\
\text { model }\end{array}$ & $\begin{array}{l}\text { Radiologic } \\
\text { grouping }\end{array}$ & 0.181 & $\begin{array}{c}0.575 \\
(0.457 \\
0.693)\end{array}$ & $16.1 \%$ & $98.9 \%$ & $71.4 \%$ & $87.5 \%$ & $87.0 \%$ & 0.150 \\
\hline $\begin{array}{l}\text { Conventional } \\
\text { logistic } \\
\text { model }\end{array}$ & $\begin{array}{l}\text { ALC, CRP, } \\
\text { LDH, } \\
\text { Radiologic } \\
\text { grouping }\end{array}$ & 0.181 & $\begin{array}{c}0.575 \\
(0.457 \\
0.693)\end{array}$ & $16.1 \%$ & $98.9 \%$ & $71.4 \%$ & $87.5 \%$ & $87.0 \%$ & 0.150 \\
\hline $\begin{array}{l}\text { Conventional } \\
\text { logistic } \\
\text { model }\end{array}$ & $\begin{array}{l}\text { Fever } \\
\text { profiles* }\end{array}$ & 0.010 & $\begin{array}{r}0.645 \\
(0.525 \\
0.765)\end{array}$ & $32.3 \%$ & $96.7 \%$ & $62.5 \%$ & $89.4 \%$ & $87.4 \%$ & 0.290 \\
\hline $\begin{array}{l}\text { Conventional } \\
\text { logistic } \\
\text { model }\end{array}$ & $\begin{array}{l}\text { Fever } \\
\text { profiles*, } \\
\text { Radiologic } \\
\text { grouping }\end{array}$ & 0.019 & $\begin{array}{c}0.632 \\
(0.512 \\
0.751)\end{array}$ & $29.0 \%$ & $97.3 \%$ & $64.3 \%$ & $89.1 \%$ & $87.4 \%$ & 0.263 \\
\hline $\begin{array}{l}\text { Deep neural } \\
\text { network }\end{array}$ & $\begin{array}{l}12 \\
\text { sequential } \\
\text { fever data }\end{array}$ & $<0.001$ & $\begin{array}{c}0.803 \\
(0.699 \\
0.908)\end{array}$ & $64.5 \%$ & $96.2 \%$ & $74.1 \%$ & $94.1 \%$ & $91.6 \%$ & 0.607 \\
\hline
\end{tabular}

\section{Results}

\section{Baseline characteristics}

Overall, 716 patients with M. pneumoniae were enrolled during the five-year study period after applying the exclusion criteria. The mean age of the entire cohort was 5.6 years (range, 1-16 years), and 350 patients (48.8\%) were boys. No patients were transferred to the intensive care unit or received mechanical ventilation. One hundred sixty-three patients $(32.5 \%)$ in the training cohort $(n=501)$ and 79 patients $(36.7 \%)$ in the test cohort $(n=215)$ were classified as RMPP-3 (Figure 1$)$. Sixty-five patients $(13.0 \%)$ in the training cohort and 31 patients (14.4\%) in the test cohort were classified as RMPP-5. 
In the training cohort for RMPP-3, the duration of fever after admission and the total duration of hospitalization were significantly longer in the RMPP-3 group $(p<0.001)$ than in the non-RMPP-3 group (Table 1). The mean WBC count, percentage of neutrophils, percentage of lymphocytes, platelets, CRP, LDH, radiologic grouping, and presence of pleural effusion were significantly different in the RMPP-3 group $(p<0.001)$. Using these variables from the univariate analysis, a conventional logistic model predicting RMPP-3 was created, which included platelets (odds ratio (OR) 0.991, $p<0.001$ ), CRP (OR $1.014, p<0.001)$, LDH (OR 1.006, $p<0.001)$, and radiologic grouping $(p<0.001)$ as significant risk factors (Table 3).

\section{Model development for predicting RMPP-5 from the training cohort}

In the training cohort for RMPP-5, the duration of fever after admission and the total duration of hospitalization were significantly longer in the RMPP- 5 group $(p<0.001)$ than in the non-RMPP- 5 group (Table 2). The mean WBC count, percentage of neutrophils, lymphocyte counts, platelets, CRP, LDH, and radiologic grouping, presence of pleural effusion, fever profiles, and 12 sequential body temperatures were significantly different in the RMPP-5 group $(p<0.001)$. With these variables, a conventional logistic model predicting RMPP-5 was created, and it included radiologic grouping (OR 1.693, group 2 vs. 1; OR 3.457, group 3 vs. 1; OR 13.947, group 4 vs. $1 ; p=0.007$ ), lowest body temperature (OR 6.494, $p=0.001$ ), and frequency of peak fever within 48 hours (OR 1.603, $p=0.001$ ) as significant risk factors (Table 4 ).

Including two hidden layers (128 neurons in the first layer and 64 neurons in the second layer), a DNN model was created using 12 sequential body temperatures. The validation loss and validation accuracy of the DNN model were 0.1807 and 0.9172 , respectively (epoch $=15$, Figure 2 ).

\section{Prediction of RMPP-3 in the test cohort}

The performance of conventional logistic models predicting RMPP-3 is compared in Table 5. Among the prediction models using individual variables, the prediction model using radiologic grouping showed the best values (area under the curve (AUC) 0.725 , sensitivity $63.3 \%$, and specificity $81.6 \%$ ). The prediction power of the model was further increased by the addition of platelets, CRP, and LDH (AUC 0.775, sensitivity $64.6 \%$, and specificity $90.4 \%$ ) as laboratory variables (Table 5 , Figure 3 ).

\section{Prediction of RMPP-5 in the test cohort}

The performance of conventional logistic models predicting RMPP-5 is compared in Table 6. While conventional logistic models using only radiological grouping did not show significant predictive power in the test cohort, prediction models using the fever profiles (lowest temperature and frequencies of peak fever) showed significant predictive power, although with a sensitivity of $32.3 \%$ and a specificity of $96.7 \%$ in the test cohort (AUC 0.645, $p=0.010$ ). However, the DNN model using data of 12 sequential body temperatures demonstrated a better and significant outcome (sensitivity $64.5 \%$ and specificity $96.2 \%$, AUC 0.803, $p<0.001$ ) (Figure 4). 


\section{Discussion}

To prevent the progression of MP pneumonia resulting in severe and prolonged clinical course, early recognition and timely treatment is important for patients who display clinical and radiological aggravation during macrolide therapy. ${ }^{8,11-13}$ To our knowledge, this is the first study to demonstrate a prediction model for refractory MP pneumonia based on readily accessible sequential fever data in addition to clinical, laboratory, and radiologic variables at admission. For prediction of RMPP-3, a conventional logistic model using only radiologic grouping showed increased sensitivity $(63.3 \%)$ than the model using laboratory values, including CRP and LDH. Adding laboratory values in the prediction model using radiologic grouping did not meaningfully contribute to an increase in sensitivity (64.6\%). For the prediction of RMPP-5, laboratory values and radiologic grouping showed lower sensitivities ranging from $12.9 \%-16.1 \%$. However, prediction models using the predefined fever profiles showed significantly increased sensitivity for predicting RMPP-5, and neural network models using 12 sequential fever data showed a greatly increased sensitivity of $64.5 \%$. Predicting high-risk patients for refractory MP pneumonia would enable physicians to calibrate their expectations of progression in these patients and to provide earlier alternative treatment.

Several studies have tried to identify predictors for refractory MP pneumonia and have suggested individual cut-off values of inflammatory markers, namely CRP, LDH, and ferritin or cytokines, such as IL6, IL-8, IL-10, IL-18, and interferon-gamma. ${ }^{11,12,14-16}$ However, the application of these findings in clinical practice is limited by lower prediction power or accessibility of the tests. Although it is plausible that increased inflammatory cytokines are related to the severity of MP pneumonia, serum cytokine assays are mostly limited for research purposes and are not routinely measured. Bronchoscopy and bronchoalveolar lavage studies are useful tools not only for identifying the causative organism but also for the removal of mucosal plugs in severe pneumonia, but are generally performed for a small proportion of MP pneumonia cases. The requirement of sedation, the necessity of special equipment, and the need for an experienced bronchoscopist limit their accessibility.

A study using CRP value of $16.5 \mathrm{mg} / \mathrm{L}$ as the cut-off value showed a sensitivity of $74.7 \%$ and a specificity of $77.2 \%$ for predicting refractory MP pneumonia. ${ }^{17}$ However, our prediction model created using the CRP level of the training cohort showed a sensitivity of $32.9 \%$ for the prediction of RMPP-3 in the test cohort even when it was combined with the LDH level (Table 5). For the prediction of RMPP-5, our prediction model using the CRP level showed a lower sensitivity of $12.9 \%$ even when used in combination with ALC and LDH levels (Table 6). Previous prediction models, created without validation, are inevitably vulnerable to model overfitting, resulting from institutional selection bias, which limits their clinical use. Therefore, a reasonable prediction model should undergo internal validation by a separate test cohort or external validation using data from another institution. Thus, it is understandable that previously identified laboratory markers such as CRP and LDH showed lower sensitivities (below 30\%) for predicting RMPP-3 and RMPP-5 in our cohorts (Tables 5 and 6). Such low sensitivities limit their clinical application for the timely detection of refractory MP pneumonia. To overcome such bias, our 716 enrolled patients were 
divided into training and test datasets for internal validation, which prevented overfitting and created a reasonable prediction model.

For prediction of RMPP-3, according to a previous study, initial radiologic grouping was the most prominent predictor. ${ }^{18}$ While the underlying mechanisms are still not clear, the pattern of pulmonary lesions in MP infection is reported to be influenced by the characteristics of host cell-mediated immunity. ${ }^{19,20}$ Thus, radiological evidence of lung involvement is consistent with the strong host immune response in RMPP.

Both initial laboratory values and radiologic grouping showed limited prediction power for the prediction of RMPP-5. However, we tried to predict RMPP using initially available data and focused on the fever data during the initial 48-hour period. Inflammatory cytokines involved in the immunopathogenesis of MP infection are reported to be increased in RMPP. ${ }^{3,21,22}$ Since these cytokines act as endogenous pyrogens that play a pivotal role in inducing fever response, their levels are associated with core body temperature. ${ }^{23}$ Although initial single time-point data were limited for predicting RMPP-5, the prediction model using predefined fever profiles showed a two-fold increase in sensitivity (16.1\% to $32.3 \%$ ), and the DNN model using all 12 sequential fever data within 48 hours showed a four-fold increase in sensitivity (64.5\%) for predicting RMPP-5. Theoretically, DNN is a black-box approach, and the causes of superior prediction power of the DNN model cannot be identified. However, the greatly increased sensitivity for predicting RMPP-5 with the DNN model using only the initial 48-hour fever data is noteworthy.

The main limitation of our study is its retrospective design based on a limited number of inpatients from a single center, which might have introduced a selection bias. However, our prediction models underwent internal validation. Prediction models were created only from the data in the training cohort, and their prediction power was estimated in the test cohort, which was not used for model development.

Nevertheless, external validation of our model in a prospective, large-scale cohort is needed for validating our results. Second, prediction models were not developed using tests, namely cytokines or FOB, which were reported to be significant. We especially focused on the accessibility of the tests, and those tests were not considered useful in usual clinical practice. Third, data on macrolide resistance were not included. Although febrile days during macrolide administration were reported to be greater in macrolideresistant patients (3.5-4.0 days vs. 1.0-1.5 days), ${ }^{24,25}$ prolonged fever in RMPP patients may not imply macrolide resistance because fever might have resolved spontaneously in some macrolide-resistant patients. The clinical efficacy of macrolide for treating MP infection may not only reflect its direct antimicrobial activity but also reflect its anti-inflammatory effects. ${ }^{26}$

Development of tests based on data obtained from routine examination of vital signs and its integration into the clinical workflow can be more effective than utilizing new tests that are less verified and less accessible. Further studies utilizing such potential data are needed for improving the prediction power.

\section{Conclusions}


In summary, our study showed that for prediction of RMPP-3, a conventional logistic model using only radiologic grouping showed a favorable predictive power than the model using initial laboratory values. In contrast, RMPP-5 could not be effectively predicted using the initial laboratory and radiologic data, which were previously reported to be significantly predictive. However, the prediction models using predefined fever profiles showed a two-fold increase in sensitivity (16.1\% to $32.3 \%)$, and the DNN model using all 12 sequential fever data within 48 hours showed a four-fold increase in sensitivity (64.5\%). Further studies using more advanced mathematical models based on easily accessible large-sized clinical data are anticipated to be helpful for predicting RMPP.

\section{List Of Abbreviations}

MP: Mycoplasma pneumonia

RMPP: Refractory Mycoplasma pneumoniae pneumonia

NPA: Nasopharyngeal aspirate

PCR: Polymerase chain reaction

CBC: Complete blood count

ESR: Erythrocyte sedimentation rate

CRP: C-reactive protein

LDH: Lactate dehydrogenase

DNN: Deep neural network

ROC: Receiver operating characteristic

OR: Odds ratio

AUC: Area under the curve

FOB: Fiberoptic bronchoscopy

\section{Declarations}

\section{Ethical approval and consent to participate}

The study was designed and conducted using the format recommended by the Strengthening the Reporting of Observational Studies in Epidemiology (STROBE) guidelines. The study protocol was approved by the institutional review board of Kangdong Sacred Heart Hospital (reference number: 202011-007). The review board waived the requirement for informed consent for this study. 


\section{Consent for publication}

Not applicable

\section{Availability of data and materials}

The datasets used and/or analysed during the current study are available from the corresponding author on reasonable request.

\section{Competing interests}

The authors declare that they have no competing interests.

\section{Funding}

No funding was received for this study.

\section{Authors' contributions}

JK conceptualized and designed the study and reviewed and revised the manuscript.

MJ and BK collected data, performed the initial analysis, and drafted the initial manuscript.

All authors approved the final manuscript as submitted and agreed to be accountable for all aspects of the work.

\section{Acknowledgements}

We thank Dr. Yoon and Dr. Lim, radiologists at our institution, for reviewing the images in the manuscript.

\section{References}

1. Kannan TR, Hardy RD, Coalson JJ, et al. Fatal outcomes in family transmission of Mycoplasma pneumoniae. Clin Infect Dis. 2011;54(2):225-231.

2. Radisic M, Torn A, Gutierrez P, Defranchi HA, Pardo P. Severe acute lung injury caused by Mycoplasma pneumoniae: potential role for steroid pulses in treatment. Clin Infect Dis. 2000;31(6):1507-1511.

3. Waites KB, Talkington DF. Mycoplasma pneumoniae and its role as a human pathogen. Clin Microbiol Rev. 2004;17(4):697-728.

4. Lee E, Cho H-J, Hong S-J, Lee J, Sung H, Yu J. Prevalence and clinical manifestations of macrolide resistant Mycoplasma pneumoniae pneumonia in Korean children. Clin Exp Pediatr. 2017;60(5):151157.

5. Hong KB, Choi EH, Lee HJ, et al. Macrolide resistance of Mycoplasma pneumoniae, South Korea, 2000-2011. Emerg Infect Dis. 2013;19(8):1281. 
6. Kawai Y, Miyashita N, Kubo M, et al. Nationwide surveillance of macrolide-resistant Mycoplasma pneumoniae infection in pediatric patients. Antimicrob Agents Chemother. 2013;57(8):4046-4049.

7. Zhao F, Liu G, Wu J, et al. Surveillance of macrolide-resistant Mycoplasma pneumoniae in Beijing, China, from 2008 to 2012. Antimicrob Agents Chemother. 2013;57(3):1521-1523.

8. Tamura A, Matsubara K, Tanaka T, Nigami H, Yura K, Fukaya T. Methylprednisolone pulse therapy for refractory Mycoplasma pneumoniae pneumonia in children. J Infect. 2008;57(3):223-228.

9. Suzuki S, Yamazaki T, Narita M, et al. Clinical evaluation of macrolide-resistant Mycoplasma pneumoniae. Antimicrob Agents Chemother. 2006;50(2):709-712.

10. Waites KB, Talkington DF. Mycoplasma pneumoniae and its role as a human pathogen. Clin Microbiol Rev. 2004;17(4):697-728.

11. Oishi T, Narita M, Matsui K, et al. Clinical implications of interleukin-18 levels in pediatric patients with Mycoplasma pneumoniae pneumonia. J Infect Chemother. 2011;17(6):803-806.

12. Inamura N, Miyashita N, Hasegawa S, et al. Management of refractory Mycoplasma pneumoniae pneumonia: utility of measuring serum lactate dehydrogenase level. $J$ Infect Chemother. 2014;20(4):270-273.

13. Tagliabue $\mathrm{C}$, Salvatore $\mathrm{CM}$, Techasaensiri $\mathrm{C}$, et al. The impact of steroids given with macrolide therapy on experimental Mycoplasma pneumoniae respiratory infection. $J$ Infect Dis. 2008;198(8):1180-1188.

14. Miyashita N, Kawai Y, Inamura N, et al. Setting a standard for the initiation of steroid therapy in refractory or severe Mycoplasma pneumoniae pneumonia in adolescents and adults. $J$ Infect Chemother. 2015;21(3):153-160.

15. Choi Y-J, Jeon J-H, Oh J-W. Critical combination of initial markers for predicting refractory Mycoplasma pneumoniae pneumonia in children: a case control study. Respir Res. 2019;20(1):193.

16. Wang L, Lu S, Feng Z, et al. The early examination of combined serum and imaging data under flexible fiberoptic bronchoscopy as a novel predictor for refractory Mycoplasma pneumoniae pneumonia diagnosis. Medicine. 2017;96(50):e9364.

17. Zhang Y, Zhou Y, Li S, Yang D, Wu X, Chen Z. The clinical characteristics and predictors of refractory Mycoplasma pneumoniae pneumonia in children. PLoS One. 2016;11(5):e0156465.

18. Yoon IA, Hong KB, Lee HJ, et al. Radiologic findings as a determinant and no effect of macrolide resistance on clinical course of Mycoplasma pneumoniae pneumonia. BMC Infect Dis. 2017;17(1):402.

19. Tanaka H, Koba H, Honma S, Sugaya F, Abe S. Relationships between radiological pattern and cellmediated immune response in Mycoplasma pneumoniae pneumonia. Eur Respir J. 1996;9(4):669672.

20. Tanaka H. Correlation between radiological and pathological findings in patients with Mycoplasma pneumoniae pneumonia. Front Microbiol. 2016;7:695. 
21. Zhang Y, Mei S, Zhou Y, Huang M, Dong G, Chen Z. Cytokines as the good predictors of refractory Mycoplasma pneumoniae pneumonia in school-aged children. Sci Rep. 2016;6:37037.

22. Narita $M$, Tanaka $H$. Cytokines involved in the severe manifestations of pulmonary diseases caused by Mycoplasma pneumoniae. Pediatr Pulmonol. 2007;42:397.

23. Netea MG, Kullberg BJ, Van der Meer JWM. Circulating cytokines as mediators of fever. Clin Infect Dis. 2000;31(Supplement_5):S178-S184.

24. Matsubara K, Okada T, Matsushima T, et al. A comparative clinical study of macrolide-sensitive and macrolide-resistant Mycoplasma pneumoniae infections in pediatric patients. J Infect Chemother. 2009;15(6):380-383.

25. Suzuki S, Yamazaki T, Narita M, et al. Clinical evaluation of macrolide-resistant Mycoplasma pneumoniae. Antimicrob Agents Chemother. 2006;50(2):709-712.

26. Morozumi M, Iwata S, Hasegawa K, et al. Increased macrolide resistance of Mycoplasma pneumoniae in pediatric patients with community-acquired pneumonia. Antimicrob Agents Chemother. 2008;52(1):348-350.

\section{Figures}

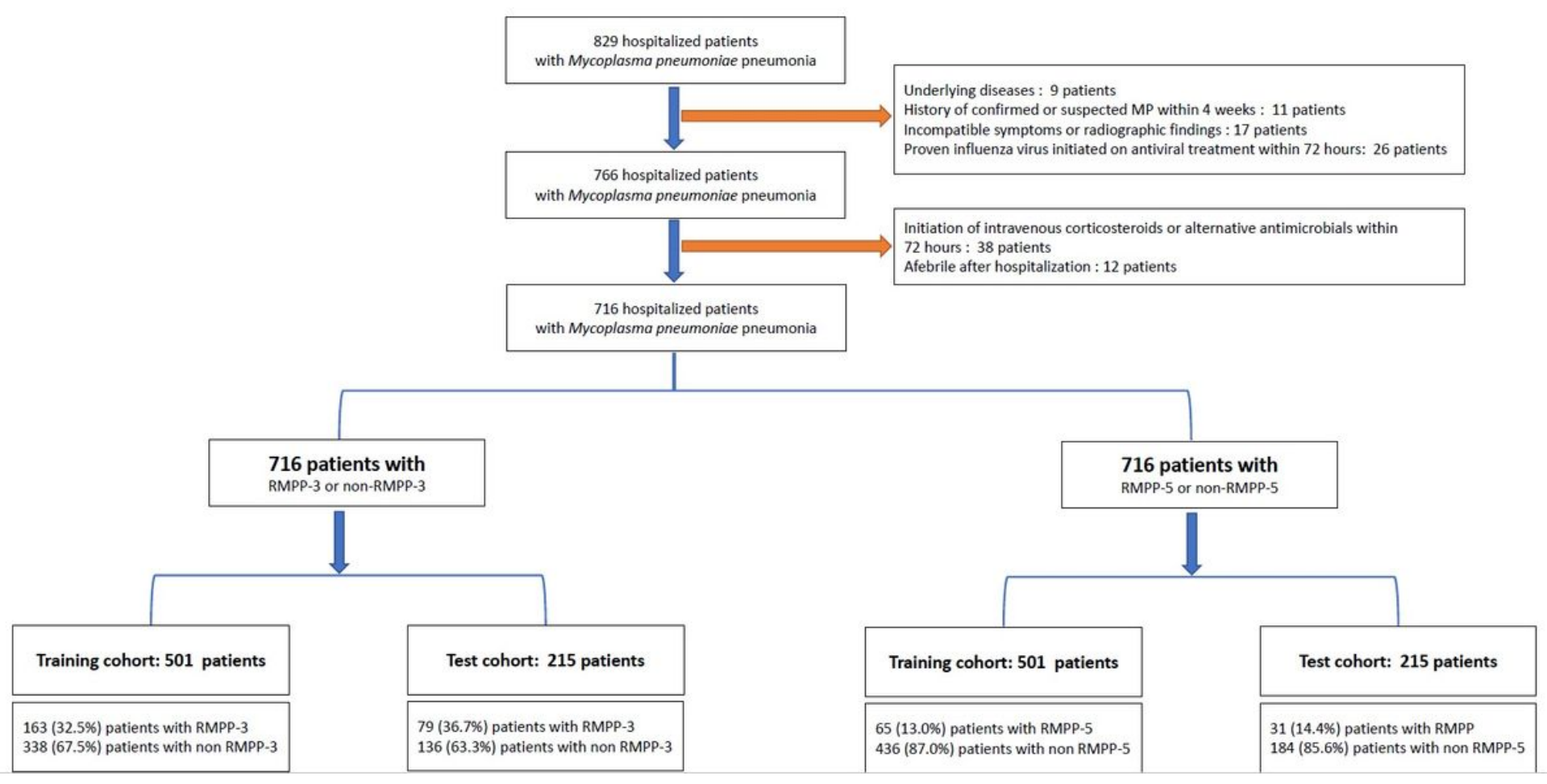

\section{Figure 1}

Study flow diagram 


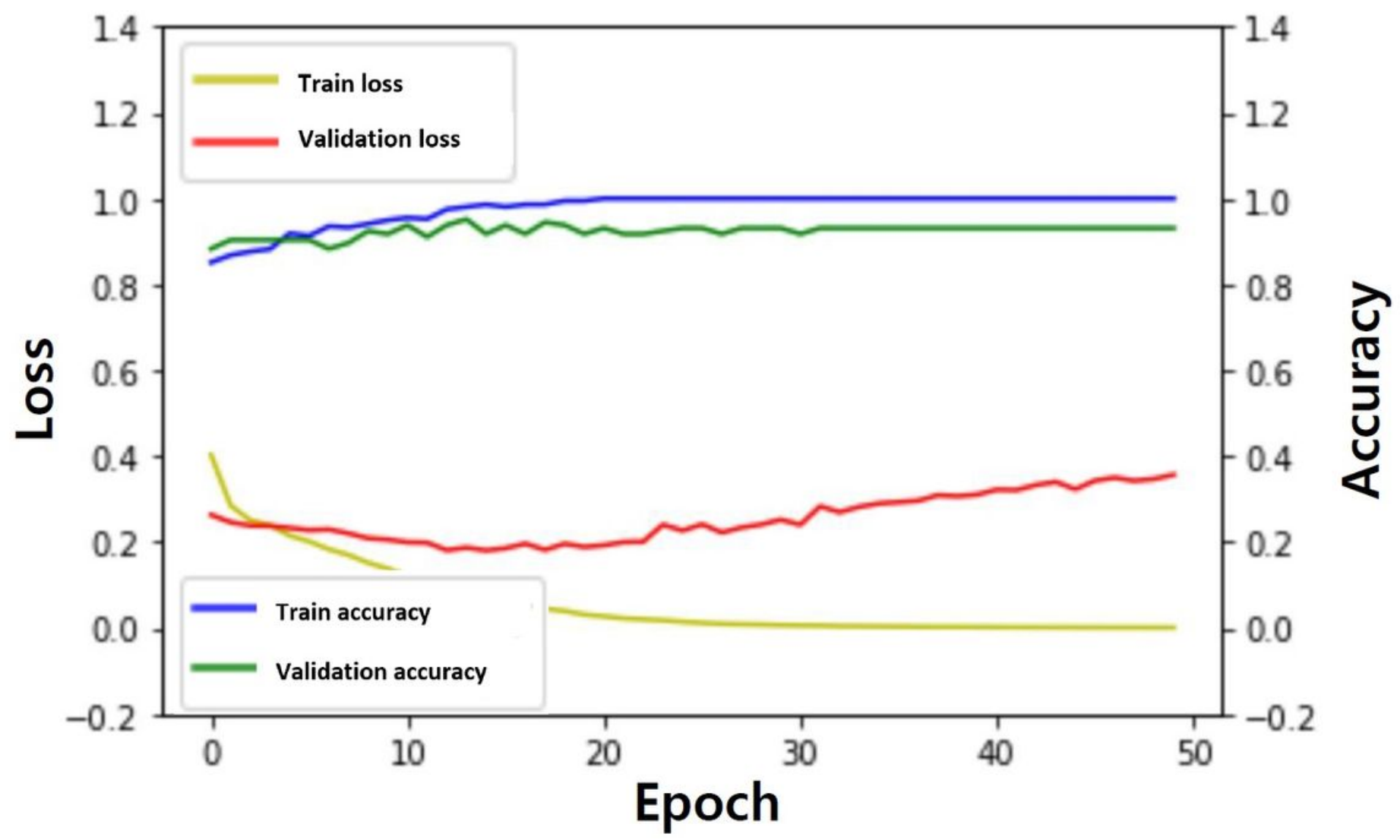

Figure 2

Loss and accuracy of the deep neural network (DNN) model 


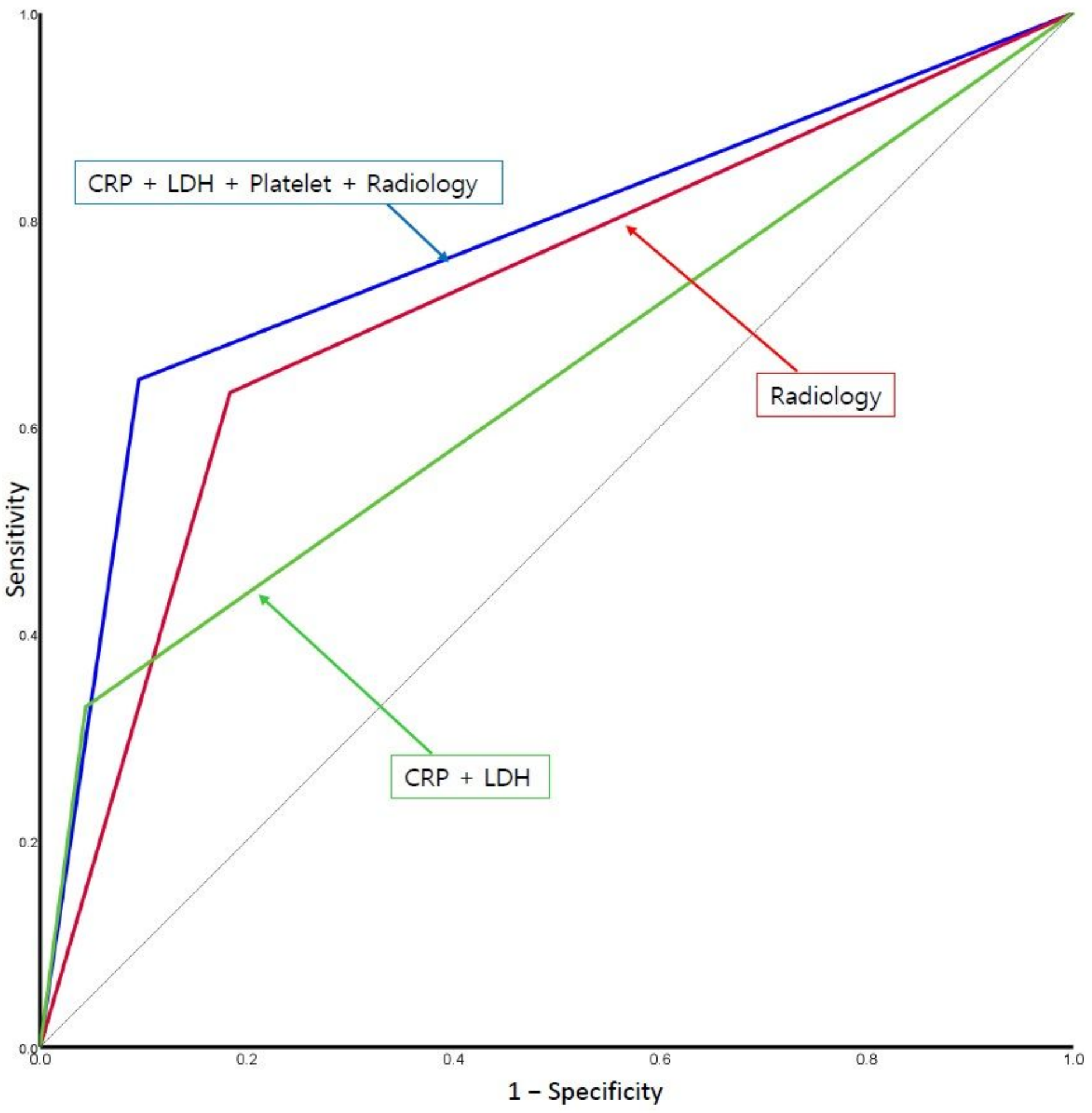

Figure 3

A receiver operating characteristic (ROC) curve for the prediction of RMPP-3 


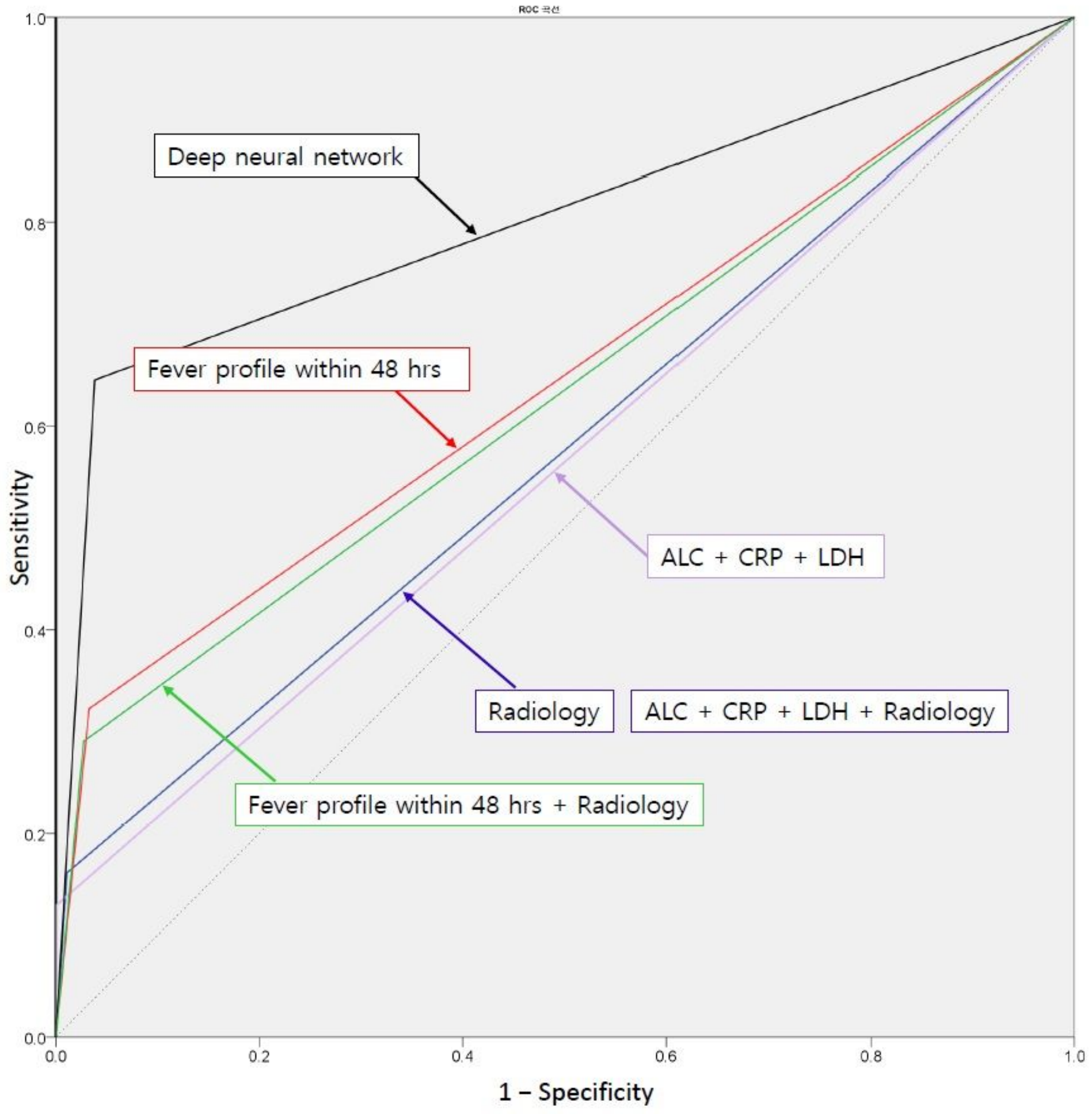

Figure 4

A receiver operating characteristic (ROC) curve for the prediction of RMPP-5 